\title{
Problematic Internet Use: Variants of Cognitive-Behavioural Model under Path Analysis
}

\author{
Chai Ping Agnes Teo ${ }^{1}$, Kokkwang Lim ${ }^{1 *}$ and Tian Po Oei ${ }^{1,2,3}$ \\ ${ }^{1}$ James Cook University, 149 Sims Drive, 387380, Singapore \\ ${ }^{2}$ Nanjing University, 22 Hankou Road, Gulou District, Nanjing, Jiangsu Province, 210093, China \\ ${ }^{3}$ University of Queensland, St Lucia QLD 4072, Australia
}

\begin{abstract}
Internet use has been receiving increasing attention from researchers and clinicians. However, few theoretical models of problematic internet use have been tested empirically, especially in Asia where a considerably high number of internet users have been documented. This study proposed variations of and tested Davis's [1] modified cognitive-behavioural model of problematic internet use in an Asian sample. The participants were 296 university students (60.8\% female, 39.2\% male) between 17 and 49 years of age in Singapore. The results from path analyses did not support Davis's model as a whole. However, significant pathways were found between psychopathology and maladaptive cognitions, as well as between maladaptive cognitions and generalised problematic internet use. Implications for research foci in the future were discussed.
\end{abstract}

\section{Publication History:}

Received: March 10, 2016

Accepted: August 01, 2017

Published: August 03, 2017

\section{Keywords:}

Computer and human behaviour, Internet use, Maladaptive cognitions, Cognitive-behavioural model, University students in Asia

\section{Introduction}

The increase in internet users over the years has aroused rising concerns with regards to problematic internet use (PIU) $[2,3,4]$ and its harmful effects associated with significant impairments in various aspects of the users' lives, including their behavioural, cognitive, emotional, social, and occupational functioning (e.g., increased mood disturbance and decreased productivity). The term PIU is selected to refer to an excessive use of the internet in a manner that "involves fewer theoretical overtones than terms such as "internet addiction" [2]. In contrast to studies in Western societies (e.g., Finland, Greece, the United States), those conducted in Asian countries (e.g., China, Korea, Taiwan) reported higher prevalence rates of PIU [5]. In particular, it was suggested in DSM-5 [6] that environmental background, particularly in Asia, might be a potential risk factor for the development for PIU. In 2014, 42.3\% of the population in the world were internet users and the majority of reported internet users (45.7\%) were from Asia [7], which showed the highest prevalence rate of PIU globally [6]. Currently, internet users in Asia have further become the majority of internet users worldwide [7]. However, as PIU is currently listed under a condition requiring further investigation, it may be premature to make any firm conclusions regarding the relationship between PIU and Asia.

Aside from the apparently substantial relationship between the Asian environment and PIU, an important issue to be further examined is the absence of a demonstrably working theoretical model for PIU in general. While there have been theoretical models proposed for etiological processes for PIU, relatively limited studies have been conducted to test these models $[1,8,9]$. For instance, Caplan's [8] model, which was based on Davis's [1] cognitive-behavioural approach to PIU, posited that individuals with psychosocial issues such as depression and loneliness would exhibit a preference for online social communication as compared to face-to-face communication. This preference for online communication would then lead to excessive overuse of the internet, which would, in turn, exacerbate their offline or "real-life" problems.

Among the different approaches to PIU proposed, Davis [1] sited one of the earliest theoretical models of PIU. Davis had differentiated two sub-types of PIU: specific (SPIU) and generalised (GPIU). SPIU, as its name suggests, is described as an excessive usage of a specific function of the internet and is assumed to be the result of pre-existing psychopathology, whereas GPIU refers to a multidimensionally excessive use of the internet. Many studies [3,10-13] based on Davis's [1] model investigated only some related variables (e.g., depression) based on the original model and included some other variables (e.g., loneliness) that were not included in the original conceptualisation. Additionally, these studies had only examined relationships or partial pathways between these variables and GPIU, with contradictory findings. For example, there were studies $[10,11]$ that reported conflicting results with regards to the role of depression predicting PIU. These issues have led to questions regarding the applied value of Davis's original conceptualisation. Given that it has been nearly two decades since Davis presented this seminal framework, more needs to be done to test the model as a whole. In particular, university students have been identified as being relatively more susceptible to developing PIU [14,15]. The concept of "emerging adulthood" has been raised to refer to the developmental stage of people approximately between 18 and 25 years old who encounter certain distinctive experiences that belong to neither adolescence nor adulthood [16]. It is postulated that emerging adults face an increased risk of engaging in addictive behaviors that impact on their perceptions of themselves and relationships with others [17] as a result of their changing neurobiology and social-cultural milieu [18]. This relatively high-risk population can then benefit from model-driven studies that could help to clarify any risk factors involved.

\section{Proposed variations of Davis's cognitive-behavioural model of GPIU}

There are aspects of Davis's conceptual model [1] that have posed challenges to model testing. Firstly, situational cue/reinforcement (i.e.,the positive reinforcement experienced when using the internet)

"Corresponding Author: Dr. Kokkwang Lim, James Cook University, 149 Sims Drive, Singapore 387380; E-mail: kokkwang.lim@jcu.edu.au

Citation: Teo CPA, Lim K, Oei TP (2017) Problematic Internet Use: Variants of Cognitive-Behavioural Model under Path Analysis. Int J Psychol Behav Anal 3: 130. doi: https://doi.org/10.15344/2455-3867/2017/130

Copyright: () 2017 Teo et al. This is an open-access article distributed under the terms of the Creative Commons Attribution License, which permits unrestricted use, distribution, and reproduction in any medium, provided the original author and source are credited. 
Citation: Teo CPA, Lim K, Oei TP (2017) Problematic Internet Use: Variants of Cognitive-Behavioural Model under Path Analysis. Int J Psychol Behav Anal 3: 130. doi: https://doi.org/10.15344/2455-3867/2017/130

Page 2 of 6

is a factor in Davis's model. The actual use of the internet would interact with the user's pre-existing psychopathology and shape their subsequent maladaptive cognitions about their own internet use. While situational cue/reinforcement is an important factor, it is difficult to operationalise and measure it, because situational cue/ reinforcement and the use of the internet itself cannot be easily isolated from each other for them to be studied independently. In a natural setting "positive reinforcement" from internet use takes place during the internet use itself. In this study, the amount (i.e., hours) of internet use was taken to represent both the situational cue/reinforcement and the physical amount of internet use.

Secondly, Davis's [1] model indicated that the symptoms of GPIU were a separate factor from the presence of GPIU itself. This theoretical distinction presents a challenge to the extent that the measurement of the presence of GPIU naturally implies the presence of various symptoms or problems arising from GPIU itself. Thus, to make Davis's model to more empirically testable, this study combined these two variables into one, i.e., GPIU.

Wright [19] proposed a set of rules with respect to path tracing in order to establish a valid relationship between any two variables in a model. In particular, Wright noted that in path tracing, an arrow should not go forward and backward between the same two variables. Therefore, the arrow conceptualised by Davis [1] between GPIU and maladaptive cognitions (feedback loop) was removed for model testing. To facilitate path analysis, a major variation (with minimum variations) of Davis's cognitive-behavioural model of GPIU is shown in Figure 1.

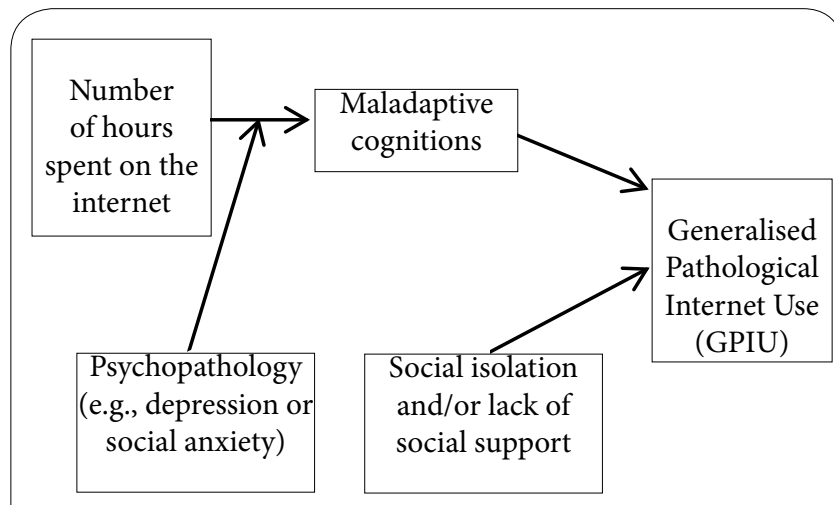

Figure 1: Proposed first variant of cognitive-behavioural model of GPIU.

\section{Aims and hypotheses}

Asia currently accounts for the highest proportion of internet users in the world. Apparently, there has been no study conducted to test the full model of Davis's [1] cognitive-behavioural model of GPIU. Many studies had tested only partial pathways of this model. What further complicates this gap in research is that these studies had produced inconsistent results. An overarching aim of this study was to develop and test three variants of Davis's general model of GPIU (Figure 1). Firstly, it was hypothesised that

1. The interaction between the number of hours on the internet and pre-existing psychopathology would be associated with maladaptive cognitions which would in turn be associated with GPIU.
1. Social support would be directly associated with GPIU Secondly, this study postulated that pre-existing psychopathology and actual internet use could each have a direct relationship with maladaptive cognitions. For this variant of Davis's model (Figure 2), it was hypothesised that.

2. Number of hours on the internet and pre-existing psychopathology would be individually associated with maladaptive cognitions which would in turn be associated with GPIU.

3. Social support would be directly associated with GPIU.

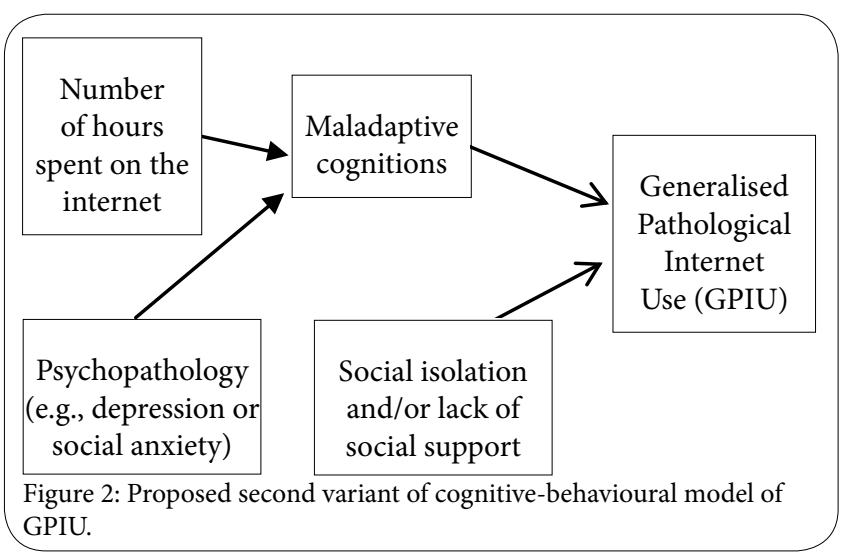

In addition to the pathways in Figure 2, a further variant of Davis's model (Figure 3) was developed for which it was hypothesised that

1. Number of hours on the internet would be directly associated with GPIU

2. Pre-existing psychopathology would be directly associated with GPIU.

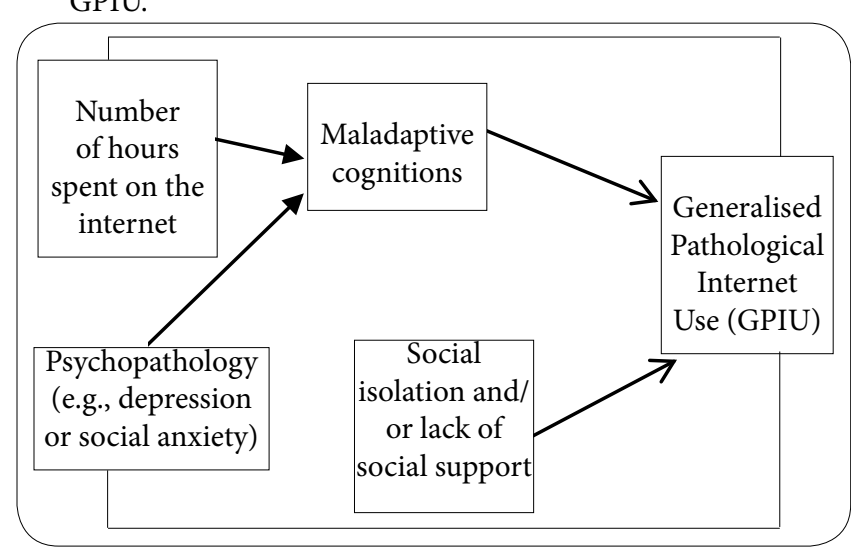

\section{Method}

\section{Participants}

The total number of the participants was 296 (180 females; $60.8 \%$ and 116 males; $39.2 \%)$ participated in this study. The mean age of participants was 22.20 years old (SD = 3.73) with $178(60.10 \%)$ identifying themselves as Chinese, $46(15.50 \%)$ as Malay, $46(15.50 \%)$ as Indian, and $26(8.90 \%)$ as of other ethnic groups.

The participants were enrolled undergraduate or postgraduate students who met two selection criteria: (1) being at least age 18 and (2) currently using the internet. They were recruited via James Cook 
University Singapore (JCUS) through direct contact and word-ofmouth communication.

\section{Measures}

The participants completed a series of questionnaires as follows:

Internet Addiction Test: The Internet Addiction Test (IAT, [20]) is a 20 -item questionnaire designed to assess the level of PIU and severity of negative consequences resulting from one's excessive internet use. The participants were asked to report their ratings on a 5-points Likert scale. The participants whose scores fell between 20 and 49 points were categorised as having mild symptoms, between 50 and 79 as endorsing moderate symptoms, and between 80 and 100 as having significant symptoms due to their internet use. The Cronbach's alpha was reported as between 0.54 to 0.82 [21], suggesting good to moderate internal consistency.

Automatic Thoughts Questionnaire: To measure maladaptive cognitions, the 30-item Automatic Thoughts Questionnaire [22] was used. The ATQ was selected as items on it matched the maladaptive cognitions mentioned by Davis [1] in his model. For example, "I am a failure" on the ATQ matched "I am a failure when I am offline" as mentioned by Davis. The ATQ was modified to be Internet-specific for this study. The participants had to indicate on a 5-points Likert scale, how frequently they had thought about each item, over the past week, as mentioned on the ATQ. The Cronbach's alpha was reported as between 0.83 to 0.93 [23], indicating good internal consistency.

Depression Anxiety Stress Scale 21: To assess psychopathology, the Depression Anxiety Stress Scale 21 (DASS-21; [24]) was used. It was designed to measure and optimally discriminate between three forms of emotional distress, namely, depression, anxiety, and stress. The participants had to rate the statements' applicability over the past week over a 4-points Likert scale. For this study, instead of differentiating between the three sub-scales, scores on the DASS- 1 were used as an overall measure of psychopathology. Likewise, the Cronbach's alpha for this scale was reported as 0.93 [24], indicating good internal consistency.

Multidimensional Scale of Perceived Social Support: The Multidimensional Scale of Perceived Social Support (MSPSS; [25]) was developed to assess an individual's perceived level of social support. It is a 12 -item scale where participants rate, on a 7-points Likert scale, the degree to which they agreed with each statement. The MSPSS consisted of three subscales, assessing perceived level of social support from family, friends, and significant others. Scores from the MSPSS were also taken as an overall measure of perceived social support across all three domains. The Cronbach's alpha was reported as 0.87 [26], indicating good internal consistency.

\section{Procedure}

The participants could choose to complete the questionnaire in two formats: print or online. On both of the versions of the questionnaire, the participants were informed about the aims of the study and that their participation was voluntary such that they could withdraw from the study at any point in time. They were assured that their responses on the questionnaire would not contain any identifying information and that their responses would be kept strictly anonymous and confidential. However, the participants who chose to enter a lucky draw were required to provide their email addresses. They were assured that this would only be used for the lucky draw and not be revealed to a third party. Contact details of the researchers of this study were also included for any enquiries about this study. After the participants have read through all the information, they were asked to express whether they would give their informed consent for this study. In addition to the measures mentioned earlier, the participants completed a series of questions related to their internet use. Some of these internet-related questions included "How old were you when you first started using the Internet?" and "Which of the following do you use to connect to the internet most often?" The participants were also requested to estimate their numbers of hours spent (per day and per week) on various internet activities. After the questionnaire was completed, the participants were given explanations via a debrief page, where they were informed about the aims of the study and contact numbers for counselling or psychological services should they experience any distress.

As an incentive, the participants were given the option of participating in the aforementioned lucky draw for a SGD 100 retail voucher reward. Eligible JCUS students were awarded credit points for their participation. However, the JCUS students who received credit points for their participation did not have the option of participating in the lucky draw.

\section{Results}

Data for this study was analysed using IBM SPSS (Version 20.0). Path analyses were used to test the models and were conducted with IBM SPSS AMOS (Version 20.0). The data collected were first screened for the presence of any missing variables or inaccurate entries. No missing or inaccurate data were detected. Thereafter, the data were screened for the presence of univariate and multivariate outliers, using Mahalanobis distance $(\mathrm{p}<.001)$ as the criterion for multivariate outliers. A total of five outliers were detected and deleted. In addition, multicollinearity and singularity were also absent, with all correlations between the variables being less than .90 .

\section{Frequency and type of internet use}

On average, the participants reported that they first started using the Internet at 10.06 -year-old $(S D=2.43)$. The Participants reported that the laptop was the device most frequently used to connect to the Internet $(83.1 \%)$, followed by smartphone $(15.9 \%)$, and tablets (1.0\%). Table 1 shows the amount of time the participants spent on various Internet activities. The top three most frequently accessed Internet functions reported by participants were Internet messages and/or chat services such as Skype and WhatsApp, social networking services (SNS) such as Facebook and Twitter, and academically related activities. Conversely, the three Internet activities least used by participants in this study were online gambling, online stock trading, and blogging.

Table 2 shows the means, standard deviations, and correlations among the variables. They reported that the average amount of time spent on the Internet per day was 9.98 hours $(\mathrm{SD}=3.24)$. Average IAT score reported by participants in this study was $49.58(\mathrm{SD}=11.82)$, which fell in the average range. Based on IAT scores, $59.46 \%(n=176)$ of the participants were categorised as average online user, $36.82 \%$ (n $=109$ ) had occasional or frequent problems due to the Internet, and $3.72 \%(n=11)$ were facing significant problems due to the Internet. Additionally, all variables were found to be significantly correlated to one another, except for the correlations between the number of daily hours spent on the Internet and (1) ATQ and (2) MSPSS. 
Citation: Teo CPA, Lim K, Oei TP (2017) Problematic Internet Use: Variants of Cognitive-Behavioural Model under Path Analysis. Int J Psychol Behav Anal 3: 130. doi: https://doi.org/10.15344/2455-3867/2017/130

Page 4 of 6

\begin{tabular}{|l|c|c|c|c|}
\hline & \multicolumn{2}{|c|}{ Hours Per Day } & \multicolumn{2}{c|}{ Hours Per Week } \\
\hline & $\mathrm{M}$ & $\mathrm{SD}$ & $\mathrm{M}$ & $\mathrm{SD}$ \\
\hline Email & 1.23 & 1.52 & 7.85 & 7.68 \\
\hline Academic related activities & 2.29 & 1.44 & 14.47 & 8.95 \\
\hline Job searching & .31 & .56 & 2.02 & 3.69 \\
\hline SNS & 2.57 & 1.86 & 16.59 & 11.18 \\
\hline Instant messages/chat & 3.72 & 2.38 & 24.70 & 15.17 \\
\hline services & & & & \\
\hline Forums/online discussion & .24 & .50 & 1.60 & 3.33 \\
groups & & & & -.00 \\
\hline Blogging & .15 & .48 & .86 & 3.26 \\
\hline Downloading & 1.54 & 1.65 & 10.07 & 10.80 \\
\hline Streaming & 1.62 & 1.34 & 10.67 & 8.85 \\
\hline Online shopping/auction & .70 & 1.15 & 4.42 & 6.48 \\
\hline Online gaming & 1.61 & 1.44 & 11.07 & 10.25 \\
\hline Web surfing & 1.54 & 1.10 & 10.12 & 6.89 \\
\hline Searching for information & 1.39 & 1.29 & 8.76 & 6.97 \\
\hline Meeting new people & .19 & .45 & 1.24 & 2.92 \\
\hline Online gambling & .00 & .06 & .02 & .29 \\
\hline Online stock trading & .26 & .44 & 1.78 & 2.93 \\
\hline Visiting adult website & & & \\
\hline Table 1 Frequency of Usage & & & & \\
\hline
\end{tabular}

Table 1: Frequency of Usage of Internet functions.

\begin{tabular}{|c|c|c|c|c|c|}
\hline & IAT & ATQ & MSPSS & DASS & Internet \\
\hline IAT & - & & & & \\
\hline ATQ & $.63^{* *}$ & - & & & \\
\hline MSPSS & $-.17^{* *}$ & $-.20^{* *}$ & - & & \\
\hline DASS & $.43^{* *}$ & $.60^{* *}$ & $-.30^{* *}$ & - & \\
\hline Internet & $.16^{* *}$ & .11 & .01 & $.19^{* *}$ & - \\
\hline M & 49.58 & 35.50 & 59.47 & 28.94 & 9.98 \\
\hline SD & 11.82 & 13.07 & 14.32 & 9.39 & 3.24 \\
\hline
\end{tabular}

Table 2: Means, Standard Deviations and Correlations among Variables.

Note. $\mathrm{N}=296$. Internet $=$ number of hours spent on the Internet per day. ${ }^{* *} \mathrm{p}<.01$

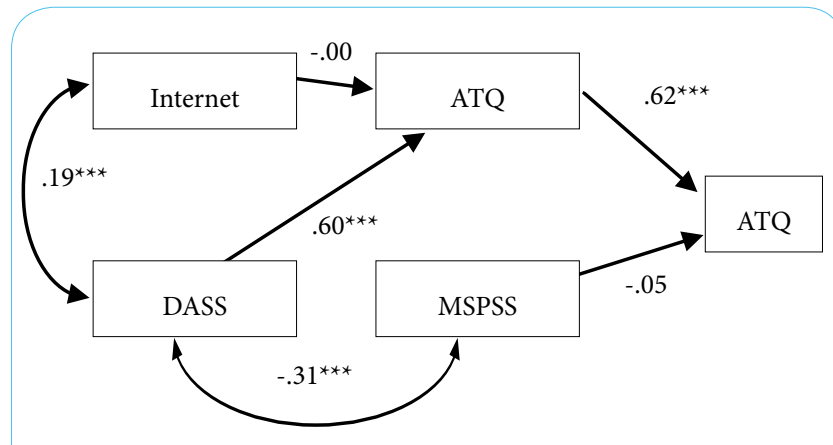

Figure 4: Path model of GPIU: Second variant. Note. ${ }^{* *} \mathrm{p}<.001$.

The first path analysis was conducted on the early model of GPIU from Davis (2001) (Figure 1). This model was rejected due to a poor fit $\left(\chi^{2}(3)=14.460, \mathrm{p}=.002, \mathrm{CFI}=.938, \mathrm{NFI}=.925, \mathrm{TLI}=.877, \mathrm{RMSEA}\right.$ $=.114, \mathrm{ECVI}=.096, \mathrm{SRMR}=.0872)$.

The second model (Figure 4) revealed a good fit, $\chi^{2}(4)=4.852$, $p$ $=.303, \mathrm{CFI}=.997, \mathrm{NFI}=.985, \mathrm{TLI}=.993, \mathrm{RMSEA}=.027, \mathrm{ECVI}=$ .091 , SRMR $=.0254$. There were only two pathways with significant regression estimates (RE): (1) the pathway between psychopathology and maladaptive cognitions $(\mathrm{RE}=.60, \mathrm{p}<.001)$ and $(2)$ the pathway between maladaptive cognitions and GPIU $(\mathrm{RE}=.62, \mathrm{p}<.001)$. Both of these pathways were in the positive direction.

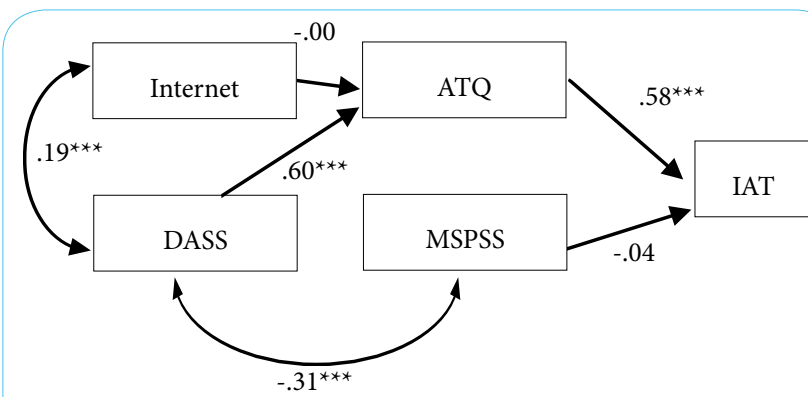

Figure 5: Path model of GPIU: Third variant.

Note. ${ }^{* *} \mathrm{p}<.001$.

\section{Discussion}

Since the introduction of Davis's [1] cognitive-behavioural model of PIU, there were numerous studies that focused on different components of this general framework [3,10-13]. This study proposed two variants to Davis's model and systematically tested them with undergraduate and postgraduate students in Asia.

Our findings showed that the three internet functions that were most commonly used were internet messages and/or chat services, SNS and academically related activities respectively. The finding that academically related activities were among one of the most commonly utilised functions of internet activities was consistent with what research has generally indicated about the university student status of the participants. Internet messages and/or chat services and SNS were expressed as the two most popular uses, reflecting pervasive utility and significance of social communication for these university-level participants.

The predominant use of the Internet for social and/or communicative purposes appeared to be in line with those found by Info-communications Development Authority of Singapore [27]. In 2012 communication activities were listed as the most (71\%) frequently accessed on the internet by Internet users aged seven and above [27]. Further, for individuals aged between 15 and 34, accessing social networks was named as the most frequent activity on mobile phones [27]. Davis [1] posited that GPIU is associated with social support and the use of social services on the internet. It may then be important that future studies investigate associations between specific types of online social/communicative services and GPIU.

By contrast, the low number of participants who used the internet for blogging purposes could be a reflection of the decline in the use blogging in favour for SNS that is observed in younger internet users [28]. 
This study found that online gambling, online stock trading, and blogging were the three least utilised Internet activities. Given the student population in this study, the low number of participants engaging in online gambling and stock trading could possibly be due to limited financial means for engaging in these activities.

\section{Findings from Path Analyses}

Of the three models tested, one model was rejected due to a poor model fit. While the other two models (Figures 4 and 5) had a good fit of model indices. Further inspection revealed that only two significant pathways, i.e., one between psychopathology and maladaptive cognitions and the other between maladaptive cognitions and GPIU.

It is important to note that the amount of internet time the majority of the participants $(59.5 \%)$ reported was in the moderate range. Further, the participants were predominately female (60.8\%). This is relevant because many studies reported associations between PIU and the male gender in Asian samples [29-31. Hence, the participants' mainly moderate use of the internet and the predominately female sample population might have restricted the number of significant relationships based on Davis's model that this study was able to detect.

While this study did not produce evidence that entirely supported Davis's (2001) cognitive-behavioural model of GPIU or its two variants, it has generated some research implications for the phenomenon of GPIU.

A conspicuous finding across all three sets of pathways was the lack of a significant pathway between perceived social support and GPIU. This seems contradictory to Davis's [1] conceptualisation and suggests that the relationship between these two variables requires a closer examination. Results of similar studies $[3,10]$ generally suggest that loneliness is a predictor of GPIU. It is then possible that the subjective experience of loneliness--in contrast to simply the perceived level of social support--can serve as a more accurate predictor of GPIU. In other words, these two variables may not be phenomenologically equivalent. Future studies could then further examine possible relationships among these tripartite variables (i.e., lack of social support, loneliness, and PIU).

The lack of a significant pathway between perceived social support and GPIU may also be related to the fairly high level of perceived social support reported by the participants $(M=59.47, S D=14.32)$. It may then still be possible that GPIU is related to the perceived social support mainly for people who experience an inordinate amount of the latter. In other words, a higher risk of engaging in GPIU could be incurred by internet users who feel substantially rather than marginally socially isolated.

In addition, this study confirms the critical pathway between maladaptive cognitions and GPIU, which is significant across all three models tested. Maladaptive cognitions are a foundational concept in Davis's [1] cognitive-behavioural model of GPIU. The consistent presence of a significant pathway between maladaptive cognitions and GPIU highlights the importance of this contributor. Currently, relatively little is known about the association between maladaptive cognitions and GPIU. However, as noted in DSM-5 [6], GPIU appears to have many similarities with other well-researched disorders such as gambling disorder and substance use disorder. Given the wellestablished association between maladaptive cognitions and gambling and substance use disorders [32,33], it is likely that maladaptive cognitions play a crucial role in the development of GPIU as well. Hence, future variants of models of GPIU can incorporate this variable with a view to distinguishing particular GPIU-related cognitions.

The significance of maladaptive cognitions also reflects clinical utility. Findings from this study suggest that, in assisting an individual with symptoms of GPIU, it may be helpful to identify specific cognitions that are related to their Internet use. For example, Young [34] proposed a cognitive-behavioural therapy (CBT) model for PIU. While the efficacy and effectiveness of CBT for PIU [35] are still being investigated, this study's findings are in favour of CBT for PIU. In particular, since the significant relationships are captured with ATQ, which is designed for studying depression, there may be common factors that underlie GPIU and depression overall.

Finally, a significant pathway was detected between psychopathology and maladaptive cognitions which supports the cognitive-behavioural perspective on psychological disorders. This is also consistent with well-established associations among psychopathology, maladaptive cognitions, and gambling disorder $[23,36]$ as well as psychopathology, maladaptive cognitions, and substance use disorder [32,37] in the field of addiction.

In summary, findings from the path analyses conducted in this study do not provide full support for the modifications made to Davis's [1] cognitive-behavioural model of GPIU and its three variants. However, the significant pathways found between psychopathology symptoms and maladaptive cognitions as well as between maladaptive cognitions and GPIU mirror those for addictions such as gambling disorder and substance use disorder in a manner that is consistent with DSM-5 [6].

\section{Limitations}

The participants in this study exhibit characteristics that have confined the generalizability of its findings. These characteristics include a nonclinical, university student, and predominantly female sample with an overall low level of GPIU. Similarly, the retrospective and self-report nature of the questionnaires limit the objectivity of the responses obtained from the participants. The cross-sectional nature of the data collected also means that causal inferences cannot be accurately extrapolated from the findings.

\section{Conclusions}

This study appears to be the first one that has simultaneously tested most of the components of Davis's [1] cognitive-behavioural model of GPIU in an Asian context. Although findings from this study do not support the proposed variants of Davis's model of GPIU, significant pathways are revealed among psychopathology, maladaptive cognitions, and GPIU, affirming most of the existing research findings. Future studies may aim to examine these relationships and further develop psychometrically testable models of GPIU that incorporate more precise maladaptive cognitions as well as more theoretically and phenomenologically coherent operationalizations of "perceived social support" and "loneliness."

\section{Competing Interests}

The authors declare that they have no competing interests. 
Citation: Teo CPA, Lim K, Oei TP (2017) Problematic Internet Use: Variants of Cognitive-Behavioural Model under Path Analysis. Int J Psychol Behav Anal 3: 130. doi: https://doi.org/10.15344/2455-3867/2017/130

Page 6 of 6

\section{References}

1. Davis RA (2001) A cognitive-behavioral model of pathological Internet use Computers in Human Behavior 17: 187-195.

2. Beard KW, Wolf EM (2001) Modification in the proposed diagnostic criteria for Internet addiction. Cyberpsychol Behav 4: 377-383.

3. Caplan SE (2002) Problematic Internet use and psychosocial well-being Development of a theory-based cognitive-behavioral measurement instrument. Computers in Human Behavior 18: 553-575.

4. Davis RA, Flett GL, Besser A (2002) Validation of a new scale for measuring problematic Internet use: Implications for pre-employment screening. Cyberpsychol Behav 5: 331-345.

5. Kuss DJ, Van Rooij AJ, Shorter GW, Griffiths MD, Van de Mheen D (2013) Internet addiction in adolescents: Prevalence and risk factors. Computers in Human Behavior 29: 1987-1996.

6. American Psychiatric Association (2013) Diagnostic and statistical manual of mental disorders (5th ed.), Washington, DC: Author.

7. Internet World Stats (2017) Internet usage statistics: The Internet big picture.

8. Caplan SE (2003) Preference for online social interaction: A theory of problematic Internet use and psychosocial well-being. Communication Research 30: 625-648.

9. Widyanto L, Griffiths M (2006) 'Internet addiction': A critical review. International Journal of Mental Health and Addiction 4: 31-51.

10. Casale S, Fioravanti G (2011) Psychosocial correlates of Internet use among Italian students. Int J Psychol 46: 288-298.

11. Durak M, Senol-Durak E (2013) Associations of social anxiety and depression with cognitions related to problematic Internet use in youths. Education and Science, 38: 19-29.

12. Pawlikowskia M, Nader IW, Burger C, Stieger S, Brand M (2014) Pathological Internet use: It is a multidimensional and not a unidimensional construct. Addiction Research \& Theory 22: 166-175.

13. Huan VS, Ang RP, Chye S (2014) Loneliness and shyness in adolescent problematic Internet users: The role of social anxiety. Child Youth Care Forum 43: 539-551.

14. Nalwa K, Anand P (2003) Internet addiction in students: A cause of concern. Cyberppsychol Behav 6: 653-656.

15. Yang SC, Tung CJ (2007) Comparison of Internet Addicts and Non-Addicts in Taiwanese High School. Computers in Human Behavior 23: 79-96.

16. Arnett JJ (2000) Emerging adulthood: A theory of development form the late teens through the early twenties. Am Psychol 55: 469-480.

17. Padilla-Walker LM, Nelson LJ, Carroll JS, Jensen AC (2010) More than just a game: Video game and internet use during emerging adulthood. J Youth Adolesc 39: 103-113.

18. Sussman A, Arnett JJ (2014) Emerging adulthood: Developmental period facilitative of the addictions. Eval Health Prof 37: 147-155

19. Wright $S$ (1934) The method of path coefficients. The Annals of Mathematical Statistics 5: 161-215.

20. Young KS (1998) Caught in the net: How to recognize the signs of Internet addiction and a winning strategy for recovery. New York: John Wiley.

21. Widyanto L, McMurran M (2004) The psychometric properties of the Internet addiction test. Cyberpsychol Behav 7: 443-450.

22. Hollon SD, Kendall PC (1980) Cognitive self-statements in depression Development of an Automatic Thoughts Questionnaire. Cognitive Therapy and Research 4: 383-395.

23. Oei TPS, Mukhtar F (2008) Exploratory and confirmatory factor validation and psychometric properties of the automatic thoughts questionnaire for Malays (ATQ-Malay) in Malaysia. Hong Kong Journal of Psychiatry 18: 92100.

24. Lovibond SH, Lovibond PF (1995) Manual for the depression anxiety stress scales (2nd ed.) Sydney: Psychology Foundation.

25. Zimet GD, Dahlem NW, Zimet SG, Farley GK (1988) The multidimensiona scale of perceived social support. Journal of Personality Assessment 52 $30-41$
26. Kazarian SS, McCabe SB (1991) Dimensions of social support in the MSPSS: Factorial structure, reliability, and theoretical implications. Journal of Community Psychology 19: 150-160.

27. Hu L, Bentler PM (1999) Cutoff criteria for fit indexes in covariance structure analysis: Conventional criteria versus new alternatives. Structural Equation Modeling 6: 1-55.

28. Infocomm Development Authority of Singapore (2013) Annual survey on infocomm usage in households and by individuals for 2012

29. Kopytoff VG (2011) Blogs Wane as the Young Drift to Sites Like Twitter. The New York Times.

30. Chou C, Hsiao M (2000) Internet addiction, usage, gratification, and pleasure experience: The Taiwan college students' case. Computers \& Education 35: 65-80.

31. Lam LT, Peng ZW, Mai JC, Jing J (2009) Factors associated with Internet addiction among adolescents. Cyberpsychol Behav 12: 551-555.

32. Tsai HF, Cheng SH, Yeh TL, Shih C, Chen KC, et al. (2009) The risk factors of Internet addiction: A survey of university freshmen. Psychiatry Res 167: 294-299.

33. Kirisci L, Tarter RE, Vanyukov M, Reynolds M, Habeych M (2004) Relation between cognitive distortions and neurobehavior disinhibition on the development of substance use during adolescence and substance use disorder by young adulthood: A prospective study. Drug Alcohol Depend 76: $125-133$.

34. Oei TPS, Lin J, Raylu N (2008) The relationship between gambling cognitions, psychological states, and gambling: A cross-cultural study of Chinese and Caucasians in Australia. Journal of Cross-Cultural Psychology 39: 147-161.

35. Young KS (2011) CBT-IA: The first treatment model for Internet addiction Journal of Cognitive Psychotherapy 25: 304-312.

36. Khazaal Y, Xirossavidou C, Khan R, Edel Y, Zebouni F, et al. (2012) Cognitive-Behavioral treatments for "Internet addiction". The Open Addiction Journal 19: 30-35.

37. Raylu N, Oei TPS (2002) Pathological gambling: A comprehensive review. Clinical Psychology Review 22: 1009-1061.

38. Armstrong TD, Costello EJ (2002) Community studies on adolescent substance use, abuse, or dependence and psychiatric comorbidity. J Consult Clin Psychol 70: 1224-1239. 